\title{
The Existence of Nontrivial Solutions to a Class of Quasilinear Equations
}

\author{
Xiaoyao Jia and Zhenluo Lou \\ Mathematics and Statistics School, Henan University of Science and Technology, No. 263 Kai-Yuan Road, Luo-Long District, \\ Luoyang, Henan Province 471023, China \\ Correspondence should be addressed to Zhenluo Lou; louzhenluo@amss.ac.cn
}

Received 12 March 2021; Revised 9 April 2021; Accepted 15 April 2021; Published 26 April 2021

Academic Editor: Igor E. Verbitsky

Copyright ( 2021 Xiaoyao Jia and Zhenluo Lou. This is an open access article distributed under the Creative Commons Attribution License, which permits unrestricted use, distribution, and reproduction in any medium, provided the original work is properly cited.

In this paper, we study the following quasilinear equation: $-\operatorname{div}(\phi(|\nabla u|) \nabla u)+\phi(|u|) u=f(u)$ in $\mathbb{R}^{N}$, where $\phi \in C^{1}\left(\mathbb{R}^{+}, \mathbb{R}^{+}\right)$and $\Phi(t)=\int_{0}^{t} s \phi(|s|) d s$. In the Orlicz-Sobolev space, by variational methods and a minimax theorem, we prove the equation has a nontrivial solution.

\section{Introduction}

In this paper, we consider the following quasilinear elliptic equation:

$$
-\Delta_{\Phi} u+\phi(|u|) u=f(u) \text { in } \mathbb{R}^{N}
$$

where $N \geq 3$ is the dimension of space and $\phi \in C^{1}\left(\mathbb{R}^{+}, \mathbb{R}^{+}\right)$ satisfies the following conditions:

$\left(\phi_{1}\right) \lim _{t \longrightarrow 0} \phi(t) t=0, \lim _{t \longrightarrow+\infty} \phi(t) t=+\infty, \quad(\phi(t) t)^{\prime}>0$, for $t>0$

$\left(\phi_{2}\right)$ There exist $p, q \in(1, N), q<p<q^{*} \equiv N q /(N-\mathrm{q})$, such that $q-2 \leq\left(\phi^{\prime}(t) t\right) /(\phi(t)) \leq p-2$, for $t>0$

$\Phi$ is a continuous function on $\mathbb{R}$ defined by

$$
\Phi(t)=\int_{0}^{t} \phi(s) s d s, \quad \text { for } t>0
$$

and $\Phi$-Laplace is defined by

$$
\Delta_{\Phi} u=\operatorname{div}(\phi(|\nabla u|) \nabla u) .
$$

Let

$$
\Phi_{*}^{-1}(t)=\int_{0}^{t} \frac{\Phi^{-1}(s)}{s^{(N+1) / N}} d s .
$$

The quasilinear elliptic equation is an important partial differential equation; in recent years, many researchers have studied the following equation:

$$
-\Delta_{p} u=f(x, u) \text { in } \Omega,
$$

where $\Omega \subset \mathbb{R}^{N}$ is an open set, $1<p<N$. Under some growth conditions, many people proved the existence and multiplicity of solutions to (5) and several mathematicians also obtained the bifurcation and asymptotic properties. By variational method and maximal principle, Guo [1] and GuoWebb [2] obtained the existence and uniqueness of the solution to (5) and they also considered the partial symmetric properties of the solutions. Guedda-Veron [3] used topology and spectrum analysis to study the bifurcation and multiplicity of the solutions. We point out that by constructing the pseudogradient vectors of $p$-Laplace operator, Zhang-Li [4] firstly obtained the sign-changing solutions to (5) and see also Zhang-Chen-Li [5], for more results of $p$-Laplace equations, one can see [6-8] and the references therein. By variational method and minimax theorem, Li-Guo [9] and 
Li-Liang [10] studied the $p-q$-Laplace equation and they obtained the existence and multiple solutions.

In [11], Franchi-Lanconelli-Serrin studied the quasilinear equation (1), and in weighted Sobolev space, they considered the existence and uniqueness of the solution to (1). In fact, the function $\phi$ is a general elliptic equation. For example, if $\phi(u) \equiv 1$, then (1) is the Laplace equation; if $\phi(u)=u^{p-2}$, then (1) is the $p$-Laplace equation; $\phi(u)=1 / \sqrt{1+p^{2}}$, then (1) is the curvature equation. The Orlicz-Sobolev space is a kind of general norm space and one can study the quasilinear equations in this space. Carvalho-Silva-Goulart [12] and Carvalho-Silva-Goncalves-Goulart [13] considered (1) in the Orlicz-Sobolev space, by variational method and concentration-compactness theorem. They obtained the existence of (1), and they also studied the famous problem, i.e., concave and convex terms.

In this paper, let $f(t)$ satisfy the following conditions:

$\left(f_{1}\right) f \in C^{1}(\mathbb{R}, \mathbb{R})$. For $t \geq 0, f(t) \geq 0$, and for $t<0, f(t)=0$

$\left(f_{2}\right) \lim _{t \rightarrow 0^{+}}(f(t) / t \phi(t))=0, \lim _{t \rightarrow+\infty}(f(t) / t \phi(t))=+\infty$

$\left(f_{3}\right)$ There exists a constant $\lambda \in(0,1)$, such that $\lim _{t \rightarrow+\infty}$ $(f(t) / g(t))=0$, with $g(t) \equiv(t \phi(t))^{\lambda}\left(\Phi_{*}{ }^{\prime}(t)\right)^{1-\lambda}$ for $t>0$

$\left(f_{4}\right)(p-1) f(t)<f^{\prime}(t) t$ for all $t>0$

$\left(f_{5}\right) f(t)$ satisfies the Ambrosetti-Rabinowitz condition: there exists a $\theta>0$ such that

$$
0 \leq F(t) \equiv \int_{0}^{t} f(s) d s \leq \frac{1}{p+\theta} f(t) t, \quad \text { for } t>0
$$

Under the preceding conditions, by a variational method, we obtain the existence of a nontrivial solution to (1). We follow the idea in [10] to obtain the existence of nontrivial solution. Our conditions $\left(f_{1}\right)-\left(f_{5}\right)$ are slightly different from what is in [10]. Conditions $\left(f_{1}\right)$ and $\left(f_{2}\right)$ are standard. Our condition $\left(f_{3}\right)$ is the improvement of $\left(f_{3}\right)$ in [10], which is used to obtain the superlinear growth of $f$ at $t \longrightarrow+\infty$. The condition $\left(f_{4}\right)$ is to guarantee that $A$ is a Finsler manifold which is used to obtain a special minimizing sequence (see Lemma 14-Lemma 16). Condition $\left(f_{5}\right)$ is the compactness condition.

Remark 1. The condition $\left(\phi_{2}\right)$ implies that for $t>0$,

$$
q \leq \frac{\phi(t) t^{2}}{\Phi(t)} \leq p
$$

Remark 2. By $\left(f_{2}\right)$ and (7), we have that

$$
\begin{aligned}
& \lim _{t \rightarrow 0^{+}} \frac{F(t)}{\Phi(t)}=\lim _{t \rightarrow 0^{+}} \frac{f(t)}{t \phi(t)}=0 ; \\
& \lim _{t \rightarrow 0^{+}} \frac{t f(t)}{\Phi(t)}=\lim _{t \rightarrow 0^{+}} \frac{t f(t)}{t^{2} \phi(t)} \frac{t^{2} \phi(t)}{\Phi(t)}=0 .
\end{aligned}
$$

This paper is organized as follows. In Section 2, we recall some results of the Orlicz-Sobolev space; in Section 3, we list and prove some preliminary results; and in Section 4, we prove our main theorem.

\section{Orlicz-Sobolev Spaces}

In this section, we recall some useful knowledge for the Orlicz space and Orlicz-Sobolev space and give some inequalities on $\Phi$. The reader can refer to $[14,15]$ for more details.

By condition $\left(\phi_{1}\right)$ and the definition of $\Phi$, the function $\Phi$ is a $N$-function (see [14] for the definition of $N$-function). The complementary of $\Phi$ is defined by

$$
\tilde{\Phi}(s)=\max _{t \geq 0}(s t-\Phi(t))
$$

for $s \geq 0$. It is easy to see that $\Phi$ and $\tilde{\Phi}$ are complementary $N$-functions. By (7) (Chapter $8[14]$ ), the function $\Phi$ and $\tilde{\Phi}$ satisfies the following inequality:

$$
\tilde{\Phi}\left(\frac{\Phi(t)}{t}\right)<\Phi(t), \quad \text { for } t>0 \text {. }
$$

By Lemma 4, Lemma 5, and Lemma 8 below, one can see that $\Phi, \tilde{\Phi}$, and $\Phi_{*}$ satisfy $\Delta_{2}$-condition (see [14] for the definition of $\Delta_{2}$-condition).

For any $\Omega \subset \mathbb{R}^{N}$, under the assumptions $\left(\phi_{1}\right)$ and $\left(\phi_{2}\right)$, the Orlicz space $L_{\Phi}(\Omega)$ contains all measurable functions $u: \Omega \longrightarrow \mathbb{R}$ which satisfy

$$
\int_{\Omega} \Phi(|u(x)|) d x<\infty
$$

and the Luxemburg norm on $L_{\Phi}(\Omega)$ is defined by

$$
\|u\|_{\Phi, \Omega}=\inf \left\{\lambda>0 \mid \int_{\Omega} \Phi\left(\frac{|u(x)|}{\lambda}\right) d x \leq 1\right\} .
$$

For any integer $m \geq 1$, the corresponding Orlicz-Sobolev space $W^{m} L_{\Phi}(\Omega)$ is defined by

$$
W^{m} L_{\Phi}(\Omega)=\left\{u \in L_{\Phi}(\Omega)\left|D^{\alpha} u \in L_{\Phi}(\Omega),\right| \alpha \mid \leq m\right\},
$$

and the norm on $W^{m} L_{\Phi}(\Omega)$ is defined by

$$
\|u\|_{m, \Phi, \Omega}=\|u\|_{\Phi, \Omega}+\sum_{|\alpha| \leq m}\left\|D^{\alpha} u\right\|_{\Phi, \Omega}
$$

Let $E_{\Phi}(\Omega)$ denote the closure in $L_{\Phi}(\Omega)$ of function $\mathrm{u}$ which are bounded on $\Omega$ and have bounded support in $\bar{\Omega}$. The space $W^{m} E_{\Phi}(\Omega)(m \in \mathbb{N}, m \geq 1)$ is defined by

$$
W^{m} E_{\Phi}(\Omega)=\left\{u \in E_{\Phi}(\Omega)\left|D^{\alpha} u \in E_{\Phi}(\Omega),\right| \alpha \mid \leq m\right\} .
$$

In the following, for simplicity, we denote $\|u\|_{\Phi, \mathbb{R}^{N}}$ and $\|u\|_{m, \Phi, \mathbb{R}^{N}}$ by $\|u\|_{\Phi}$ and $\|u\|_{m, \Phi}$. Then, the Orlicz-Sobolev space has the following properties. 
Theorem 3 (Theorem 8.20 and Theorem 8.31 [14]).

(a) The spaces $L_{\Phi}(\Omega)$ is reflexive if and only if both $(\Phi$, $\Omega)$ and $(\tilde{\Phi}, \Omega)$ are $\Delta$-regular

(b) If $(\Phi, \Omega)$ and $(\tilde{\Phi}, \Omega)$ are $\Delta$-regular, then $W^{m} E_{\Phi}(\Omega)$ $=W^{m} L_{\Phi}(\Omega)$ is reflexive

(c) $C_{0}^{\infty}\left(\mathbb{R}^{N}\right)$ is dense in $W^{m} E_{\Phi}\left(\mathbb{R}^{N}\right)$. Thus, $W^{m} E_{\Phi}\left(\mathbb{R}^{N}\right)$ $=W_{0}^{m} L_{\Phi}\left(\mathbb{R}^{N}\right)$

Since $\Phi$ and $\tilde{\Phi}$ are complementary N-functions, the following generalize Hölder inequality (see [14]) holds:

$\left|\int_{\Omega} u(x) v(x) d x\right| \leq 2\|u\|_{\Phi, \Omega}\|v\|_{\tilde{\Phi}, \Omega}, \quad$ for any $u \in L_{\Phi}(\Omega), v \in L_{\tilde{\Phi}}(\Omega)$.

Next, we recall some inequalities on $\Phi$.

Lemma 4 (Lemma $2.1[15])$. Let $\zeta_{0}(t)=\min \left\{t^{q}, t^{p}\right\}, \zeta_{1}(t)=$ $\max \left\{t^{q}, t^{p}\right\}, t \geq 0$. Then,

$$
\begin{aligned}
& \zeta_{0}(t) \Phi(\rho) \leq \Phi(\rho t) \leq \zeta_{1}(t) \Phi(\rho), \quad \text { for } \rho, t \geq 0 \\
& \zeta_{0}\left(\|u\|_{\Phi}\right) \leq \int_{\mathbb{R}^{N}} \Phi(|u|) d x \leq \zeta_{1}\left(\|u\|_{\Phi}\right), \quad \text { for } u \in L_{\Phi}\left(\mathbb{R}^{N}\right) .
\end{aligned}
$$

Lemma 5 (Lemma $2.2[15])$. Let $\zeta_{2}(t)=\min \left\{t^{q^{*}}, t^{p^{*}}\right\}, \zeta_{3}(t)$ $=\max \left\{t^{q^{*}}, t^{p^{*}}\right\}, t \geq 0$. Then,

$$
\zeta_{2}(t) \Phi_{*}(\rho) \leq \Phi_{*}(\rho t) \leq \zeta_{3}(t) \Phi_{*}(\rho), \quad \text { for } \rho, t \geq 0
$$

$$
\zeta_{2}\left(\|u\|_{\Phi_{*}}\right) \leq \int_{\mathbb{R}^{N}} \Phi_{*}(|u|) d x \leq \zeta_{3}\left(\mid u \|_{\Phi_{*}}\right), \quad \text { for } u \in L_{\Phi_{*}}\left(\mathbb{R}^{N}\right)
$$

Lemma 6 (Lemma 2.3 [15]). $\Phi(t)$ increases essentially more slowly than $\Phi_{*}(t)$ near infinity, i.e.,

$$
\lim _{t \longrightarrow+\infty} \frac{\Phi(k t)}{\Phi_{*}(t)}=0,
$$

for every constant $k>0$.

Lemma 7 (Lemma 2.4 [15]). (19) is equivalent to

$$
q^{*} \leq \frac{\Phi_{*}^{\prime}(t) t}{\Phi_{*}(t)} \leq p^{*}
$$

Lemma $8(\operatorname{Lemma} 2.5[15])$. Let $\zeta_{4}(t)=\min \left\{t^{q /(q-1)}, t^{p /(p-1)}\right\}$, $\zeta_{5}(t)=\max \left\{t^{q /(q-1)}, t^{p /(p-1)}\right\}, t \geq 0$. Then,

$$
\begin{aligned}
& \zeta_{4}(t) \tilde{\Phi}(\rho) \leq \tilde{\Phi}(\rho t) \leq \zeta_{5}(t) \tilde{\Phi}(\rho), \quad \text { for } \rho, t \geq 0 \\
& \zeta_{4}\left(\|u\|_{\tilde{\Phi}}\right) \leq \int_{\mathbb{R}^{N}} \tilde{\Phi}(|u|) d x \leq \zeta_{5}\left(\|u\|_{\tilde{\Phi}}\right), \quad \text { for } u \in L_{\tilde{\Phi}}\left(\mathbb{R}^{N}\right)
\end{aligned}
$$

We recall some imbedding results in the Orlicz-Sobolev space.

Theorem 9 (Theorem 8.12 [14]). The imbedding $L_{B}\left(\mathbb{R}^{N}\right) \subset$ $L_{A}\left(\mathbb{R}^{N}\right)$ holds if and only if $B$ dominates $A$ globally, i.e., there exists a constant $k>0$ such that $A(t) \leq B(k t)$ holds for all $t \geq 0$.

Theorem 10 (Theorem 8.36 [14]). Let $\Omega$ be an arbitrary domain in $\mathbb{R}^{N}$. If the $N$-function $\Phi(t)$ satisfies

$$
\begin{aligned}
& \int_{0}^{1} \frac{\Phi^{-1}(t)}{t^{(N+1) / N}} d t<\infty, \\
& \int_{1}^{\infty} \frac{\Phi^{-1}(t)}{t^{(N+1) / N}} d t=\infty
\end{aligned}
$$

then $W_{0}^{m} L_{\Phi}(\Omega) \subset L_{\Phi_{*}}(\Omega)$, for any integer $m \geq 1$. Moreover, if $\Omega_{0}$ is a bounded subdomain of $\Omega$, then the imbedding $W_{0}^{m} L_{\Phi}(\Omega) \subset L_{B}\left(\Omega_{0}\right)$ exists and is compact for any $N$-function $B$ increasing essentially more slowly than $\Phi_{*}$ near infinity.

Since $\Phi$ and $\tilde{\Phi}$ satisfy $\Delta_{2}$-condition, so by Theorem 3, $W^{m} L_{\Phi}\left(\mathbb{R}^{N}\right)=W_{0}^{m} L_{\Phi}\left(\mathbb{R}^{N}\right)$ is reflexive. Using Lemma 4 and Theorem 10, one can see that

$$
W^{m} L_{\Phi}\left(\mathbb{R}^{N}\right)=W_{0}^{m} L_{\Phi}\left(\mathbb{R}^{N}\right) \subset L_{\Phi_{*}}\left(\mathbb{R}^{N}\right) .
$$

By (7), (11), Lemma 4, and Lemma 8, we can see that for $u \in W^{1} L_{\Phi}\left(\mathbb{R}^{N}\right)$ and $\Omega \subset \mathbb{R}^{N}$, there exists some $C>0$ such that

$$
\begin{aligned}
\|\phi(|u|) u\|_{\tilde{\Phi}, \Omega} & \leq \zeta_{4}^{-1}\left(\int_{\Omega} \tilde{\Phi}(\phi(|u|)|u|) d x\right) \\
& \leq \zeta_{4}^{-1}\left(\int_{\Omega} \tilde{\Phi}\left(p \frac{\Phi(|u|)}{|u|}\right) d x\right) \\
& \leq C \zeta_{4}^{-1}\left(\int_{\Omega} \Phi(|u|) d x\right) \\
& \leq C \zeta_{4}^{-1}\left(\zeta_{1}\left(\|u\|_{\Phi, \Omega}\right)\right) .
\end{aligned}
$$

\section{Preliminary Results}

In this section, we prove some preliminary results for future use. 
To study the existence of solution to (1), we first study its energy functional. It is clear that the functional defining on $W^{1} L_{\Phi}\left(\mathbb{R}^{N}\right)$ is given by

$$
I(u)=\int_{\mathbb{R}^{N}} \Phi(|\nabla u|)+\Phi(|u|) d x-\int_{\mathbb{R}^{N}} F(u) d x .
$$

It is easy to see that under the assumptions $\left(f_{1}\right)-\left(f_{5}\right)$ and $\left(\phi_{1}\right)-\left(\phi_{3}\right)$, the functionals $I$ is of $C^{1}$. For any $u, v \in W^{1} L_{\Phi}\left(\mathbb{R}^{N}\right)$, we have

$\left\langle I^{\prime}(u), v\right\rangle=\int_{\mathbb{R}^{N}} \phi(|\nabla u|) \nabla u \cdot \nabla v+\phi(|u|) u v d x-\int_{\mathbb{R}^{N}} f(u) v d x$.

The $\langle\cdot, \cdot\rangle$ is the dual pairing between $W^{1} L_{\Phi}\left(\mathbb{R}^{N}\right)$ and its dual space $\left(W^{1} L_{\Phi}\left(\mathbb{R}^{N}\right)\right)^{*}$.

By condition $\left(f_{2}\right)$ and $\left(f_{3}\right)$, we have that for any $\varepsilon>0$, there exists a constant $C_{\varepsilon}>0$ such that

$$
f(t) \leq \varepsilon t \phi(t)+C_{\varepsilon} g(t), \quad \text { for } t>0 .
$$

Hence, it follows that for any $\varepsilon>0$, there exists a constant $C_{\varepsilon}>0$ such that

$$
F(t) \leq \varepsilon \Phi(t)+C_{\varepsilon} t g(t), \quad \text { for } t>0 .
$$

Lemma 11. Suppose $\left(f_{5}\right)$ holds. Then, there exists a positive constant $C>0$ such that for any $u \in \mathbb{R}^{+}, t \in \mathbb{R}^{+}$,

$$
F(t u) \begin{cases}\leq F(u) t^{p+\theta}, & \text { for } 0<t<1, \\ \geq F(u) t^{p+\theta}, & \text { for } t \geq 1 .\end{cases}
$$

Proof. By $\left(f_{5}\right)$, one has that, for $s \in \mathbb{R}^{+}$,

$$
\frac{p+\theta}{s} \leq \frac{F^{\prime}(s)}{F(s)} .
$$

Integrating the last inequality from $u$ to $t u$, we get the result in the lemma. This ends the proof.

Set

$$
A=\left\{u \in W^{1} L_{\Phi}\left(\mathbb{R}^{N}\right) \mid\left\langle I^{\prime}(u), u\right\rangle=0, u \equiv 0\right\} .
$$

Then, we show that $A$ is not an empty set.

Lemma 12. Suppose that $\left(\phi_{1}\right)-\left(\phi_{3}\right)$ and $\left(f_{1}\right)-\left(f_{5}\right)$ hold. Then, $A \neq \varnothing$.

Proof. By $\left(f_{1}\right)$ and $\left(f_{2}\right), f \equiv 0$. Hence, there exists a constant $a_{0}>0$ such that $f\left(a_{0}\right)>0$. Notice that $C_{0}^{\infty}\left(\mathbb{R}^{N}\right) \subset W^{1} L_{\Phi}$ $\left(\mathbb{R}^{N}\right)$. We choose $u_{0} \in C_{0}^{\infty}\left(\mathbb{R}^{N}\right)$ such that $u_{0} \geq 0, u_{0} \equiv 0$, and $\sup \left\{u_{0}(x) \mid x \in \mathbb{R}^{N}\right\}>a_{0}$. Let

$$
h(t)=\left\langle I^{\prime}\left(t u_{0}\right), t u_{0}\right\rangle .
$$

It is easy to show that $h(t)$ is continuous. For $t>1$, by (7), $\left(f_{5}\right)$, and Lemma 11 , we have

$$
\begin{aligned}
h(t) & =\int_{\mathbb{R}^{N}} \phi\left(t\left|\nabla u_{0}\right|\right) t^{2}\left|\nabla u_{0}\right|^{2}+\phi\left(t u_{0}\right) t^{2} u_{0}^{2} d x-\int_{\mathbb{R}^{N}} f\left(t u_{0}\right) t u_{0} d x \\
& \leq p \int_{\mathbb{R}^{N}} \Phi\left(t\left|\nabla u_{0}\right|\right)+\Phi\left(t u_{0}\right) d x-(p+\theta) \int_{\mathbb{R}^{N}} F\left(t u_{0}\right) d x \\
& \leq p t^{p} \int_{\mathbb{R}^{N}} \Phi\left(\left|\nabla u_{0}\right|\right)+\Phi\left(u_{0}\right) d x-(p+\theta) t^{p+\theta} \int_{\mathbb{R}^{N}} F\left(u_{0}\right) d x .
\end{aligned}
$$

Using the definition of $u_{0}$, one has that $\int_{\mathbb{R}^{N}} F\left(u_{0}\right) d x>0$. It follows that

$$
\lim _{t \longrightarrow+\infty} h(t)=-\infty
$$

For $0<t<1$, by (7), we have

$$
\begin{aligned}
h(t) \geq q & \int_{\mathbb{R}^{N}} \Phi\left(t\left|\nabla u_{0}\right|\right)+\Phi\left(t u_{0}\right) d x \\
& -\int_{\mathbb{R}^{N}} \frac{f\left(t u_{0}\right) t u_{0}}{\Phi\left(t u_{0}\right)} \Phi\left(t u_{0}\right) d x .
\end{aligned}
$$

By (9), we can see

$$
\lim _{t \longrightarrow 0^{+}} \frac{f\left(t u_{0}\right) t u_{0}}{\Phi\left(t u_{0}\right)}=0
$$

It follows that, for any $0<\varepsilon<1$, there exists some $\tilde{t}>0$ such that, for any $t \in(0, \tilde{t})$,

$$
\frac{f\left(t u_{0}\right) t u_{0}}{\Phi\left(t u_{0}\right)}<\varepsilon
$$

Hence,

$$
h(t) \geq(q-\epsilon) \int_{\mathbb{R}^{N}} \Phi\left(t\left|\nabla u_{0}\right|\right)+\Phi\left(t u_{0}\right) d x>0 .
$$

Therefore, there exists some $t_{0}>0$ such that $h\left(t_{0}\right)=0$. This ends the proof.

Lemma 13. For any $\epsilon>0$, there exists some $C_{\epsilon}>0$ such that

$$
\int_{\mathbb{R}^{N}}|u| g(|u|) d x \leq \epsilon \int_{\mathbb{R}^{N}} \Phi(|u|) d x+C_{\epsilon} \int_{\mathbb{R}^{N}} \Phi_{*}(|u|) d x,
$$

for any $u \in W^{1} L_{\Phi}\left(\mathbb{R}^{N}\right)$.

Proof. By Lemma 7 and (7), for $t>0$

$$
\begin{aligned}
\operatorname{tg}(t) & =\left(t^{2} \phi(t)\right)^{\lambda}\left(t \Phi_{*}{ }^{\prime}(t)\right)^{1-\lambda} \\
& \leq p^{\lambda} p^{*(1-\lambda)}(\Phi(t))^{\lambda}\left(\Phi_{*}(t)\right)^{1-\lambda} .
\end{aligned}
$$


It follows from Young's inequality that, for any $\epsilon>0$, there exists $C_{\epsilon}>0$ such that

$$
\operatorname{tg}(t) \leq \epsilon \Phi(t)+C_{\epsilon} \Phi_{*}(t)
$$

Hence, for $u \in W^{1} L_{\Phi}\left(\mathbb{R}^{N}\right)$,

$$
\int_{\mathbb{R}^{N}}|u| g(|u|) d x \leq \epsilon \int_{\mathbb{R}^{N}} \Phi(|u|) d x+C_{\epsilon} \int_{\mathbb{R}^{N}} \Phi_{*}(|u|) d x
$$

This ends the proof.

Set

$$
I_{0}=\inf \{I(u) \mid u \in A\} .
$$

Lemma 14. Suppose $(\phi 1)-(\phi 3)$ and $\left(f_{1}\right)-\left(f_{5}\right)$ hold. Then, $I_{0}>0$.

Proof. For any $u \in A$, let $T(u)=\int_{\mathbb{R}^{N}} \Phi(|\nabla u|)+\Phi(|u|) d x$. Using $(7)$ and $\left(f_{5}\right)$, we obtain that

$$
\begin{aligned}
I(u) & =T(u)-\int_{\mathbb{R}^{N}} F(u) d x \geq T(u)-\frac{1}{p+\theta} \int_{\mathbb{R}^{N}} f(u) u d x \\
& =T(u)-\frac{1}{p+\theta} \int_{\mathbb{R}^{N}} \phi(|\nabla u|)|\nabla u|^{2}+\phi(|u|) u^{2} d x \\
& \geq\left(1-\frac{p}{p+\theta}\right) T(u) \geq 0 .
\end{aligned}
$$

Hence,

$$
I_{0}=\inf \{I(u) \mid u \in A\} \geq 0
$$

By (7), (29), (30), and Lemma 13 , for any $0<\epsilon_{1}<\epsilon_{2}$, one has that there exist two positive constants $C_{\epsilon_{1}}$ and $C_{\epsilon_{2}}$ such that

$$
\begin{aligned}
I(u) & \leq \frac{1}{q} \int_{\mathbb{R}^{N}} \phi(|\nabla u|)|\nabla u|^{2}+\phi(|u|) u^{2} d x-\int_{\mathbb{R}^{N}} F(u) d x \\
& =\frac{1}{q} \int_{\mathbb{R}^{N}} f(u) u d x-\int_{\mathbb{R}^{N}} F(u) d x \\
& \leq \epsilon_{1} \int_{\mathbb{R}^{N}} \Phi(|u|) d x+C_{\epsilon_{1}} \int_{\mathbb{R}^{N}}|u| g(|u|) d x \\
& \leq \epsilon_{2} \int_{\mathbb{R}^{N}} \Phi(|u|) d x+C_{\epsilon_{2}} \int_{\mathbb{R}^{N}} \Phi_{*}(|u|) d x .
\end{aligned}
$$

Choose $\epsilon_{2}$ small enough such that $1-p /(p+\theta)-\epsilon_{2}>0$. Then, we obtain that

$$
\left(1-\frac{p}{p+\theta}-\epsilon_{2}\right) T(u) \leq C_{\epsilon_{2}} \int_{\mathbb{R}^{N}} \Phi_{*}(|u|) d x .
$$

By (25), Lemma 4, and Lemma 5, we have that

$$
\begin{aligned}
& \int_{\mathbb{R}^{N}} \Phi_{*}(|u|) d x \\
& \quad \leq \zeta_{3}\left(\|u\|_{\Phi_{*}}\right) \leq C \zeta_{3}\left(\|u\|_{1, \Phi}\right) \\
& \quad \leq C \zeta_{3}\left(\zeta_{0}^{-1}\left(\int_{\mathbb{R}^{N}} \Phi(|u|) d x\right)+\zeta_{0}^{-1}\left(\int_{\mathbb{R}^{N}} \Phi(|\nabla u|) d x\right)\right) .
\end{aligned}
$$

By the definition of $\zeta_{0}$ and $\zeta_{3}$, we have

$$
\begin{aligned}
\zeta_{3}\left(\zeta_{0}^{-1}\left(\int_{\mathbb{R}^{N}} \Phi(|u|) d x\right)+\zeta_{0}^{-1}\left(\int_{\mathbb{R}^{N}} \Phi(|\nabla u|) d x\right)\right) \\
\leq \zeta_{3}\left(\left(\int_{\mathbb{R}^{N}} \Phi(|u|) d x\right)^{1 / p}+\left(\int_{\mathbb{R}^{N}} \Phi(|u|) d x\right)^{1 / q}\right. \\
\left.\quad+\left(\int_{\mathbb{R}^{N}} \Phi(|\nabla u|) d x\right)^{1 / p}+\left(\int_{\mathbb{R}^{N}} \Phi(|\nabla u|) d x\right)^{1 / q}\right) \\
\leq C \zeta_{3}\left((T(u))^{1 / p}+(T(u))^{1 / q}\right) \\
\leq C \max \left\{(T(u))^{p * / p}+(T(u))^{p * / q},(T(u))^{q * / p}+(T(u))^{q * / q}\right\} .
\end{aligned}
$$

Combining (49)-(51), there exists a positive constant $C_{\epsilon_{2}}^{\prime}>0$, such that

$$
\begin{aligned}
\max & \left\{(T(u))^{p * / p-1}+(T(u))^{p * / q-1},(T(u))^{q * / p-1}+(T(u))^{q * / q-1}\right\} \\
\geq & C_{\epsilon_{2}}^{\prime}\left(1-\frac{p}{p+\theta}-\epsilon_{2}\right),
\end{aligned}
$$

which means $T(u)>C$ for some $C>0$. It follows that $I_{0}>$ $(1-p /(p+\theta)) C$. This completes the proof.

$$
\begin{aligned}
& \text { For } u \in W^{1} L_{\Phi}\left(\mathbb{R}^{N}\right) \text {, set } \\
& \begin{aligned}
H(u) & =\left\langle I^{\prime}(u), u\right\rangle \\
& =\int_{\mathbb{R}^{N}} \phi(|\nabla u|)|\nabla u|^{2}+\phi(|u|) u^{2} d x-\int_{\mathbb{R}^{N}} f(u) u d x .
\end{aligned}
\end{aligned}
$$

It is easy to see that $H$ is a $C^{1}$-functional. For any $u \in A$, one has that $u^{+} \equiv 0$. If $u^{+} \equiv 0$, then $f(u) \equiv 0$ by $\left(f_{1}\right)$. Noting that $u \equiv 0$, one has that $H(u)>0$, which contradicts with $u \in$ A. By $\left(\phi_{2}\right)$ and $\left(f_{4}\right)$

$$
\begin{aligned}
& \left\langle H^{\prime}(u), u\right\rangle \\
& \quad=\int_{\mathbb{R}^{N}} \phi^{\prime}(|\nabla u|)|\nabla u|^{3}+2 \phi(|\nabla u|)|\nabla u|^{2} \\
& \quad+\phi^{\prime}(|u|)|u|^{3}+2 \phi(|u|) u^{2} d x-\int_{\mathbb{R}^{N}} f(u) u+f^{\prime}(u) u^{2} d x \\
& \leq p \int_{\mathbb{R}^{N}} \phi(|\nabla u|)|\nabla u|^{2}+\phi(|u|) u^{2} d x-\int_{\mathbb{R}^{N}} f(u) u+f^{\prime}(u) u^{2} d x \\
& =\int_{\mathbb{R}^{N}}\left((p-1) f\left(u^{+}\right)-f^{\prime}\left(u^{+}\right) u^{+}\right) u^{+} d x<0 .
\end{aligned}
$$


Hence, $A$ is a closed and a complete submanifold of $W^{1}$ $L_{\Phi}\left(\mathbb{R}^{N}\right)$ with the natural Finsler structure (see [16]). Using Lemma 2.14 [10] with $n=1, e_{1}=u /\left\langle G^{\prime}(u), u\right\rangle$, one has the following.

Lemma 15. For any $u \in A, v \in W^{1} L_{\Phi}\left(\mathbb{R}^{N}\right)$, we have

$$
\left\langle\left. d I\right|_{A}(u), \pi v\right\rangle=\langle I(u), v\rangle,
$$

with $\pi$ is the projection from $W^{1} L_{\Phi}\left(\mathbb{R}^{N}\right)$ to $T_{u} A$.

By Lemma $12, A$ is not empty, and by Lemma $14, I$ is bounded from below. Hence, by Lemma 2.15 of [10] and Lemma 15, we can get the following result.

Lemma 16. There exists a sequence $\left\{u_{n}\right\} \subset A$, such that,

$$
\lim _{n \longrightarrow \infty} I\left(u_{n}\right)=I_{0} \text { and } \lim _{n \longrightarrow \infty} I^{\prime}\left(u_{n}\right)=0 \text { in }\left(W^{1} L_{\Phi}\left(\mathbb{R}^{N}\right)\right)^{*} .
$$

Lemma 17. Let $\left\{\rho_{n}\right\} \subset L^{1}\left(\mathbb{R}^{N}\right)$ be a bounded sequence and $\rho_{n} \geq 0$, then there exists a subsequence, still denoted by $\left\{\rho_{n}\right\}$, such that one of the following two possibilities occurs:

(1) (Vanishing): $\lim _{n \longrightarrow \infty} \sup _{y \in \mathbb{R}^{N}} \int_{y+B_{R}} \rho_{n}(x) d x=0$ for all $0<R$ $<+\infty$

(2) (Nonvanishing): there exists $\alpha>0,0<R<+\infty$ and $\left\{y_{n}\right\} \subset \mathbb{R}^{N}$, such that $\lim _{n \longrightarrow \infty} \int_{y_{n}+B_{R}} \rho_{n}(x) d x \geq \alpha>0$

Lemma 18. Suppose $\left\{u_{n}\right\}$ is a bounded sequence in $W^{1} L_{\Phi}$ $\left(\mathbb{R}^{N}\right)$, and for some $r>0$,

$$
\lim _{n \longrightarrow+\infty} \sup _{y \in \mathbb{R}^{N}} \int_{y+B_{r}} \Phi\left(\left|u_{n}\right|\right) d x=0
$$

Then, $\lim _{n \longrightarrow \infty} \int_{\mathbb{R}^{N}} g\left(\left|u_{n}\right|\right)\left|u_{n}\right| d x=0$.

Proof. By Lemma 7, (7), and Hölder inequality, for any $\tau \epsilon$ $(0,1)$,

$$
\begin{aligned}
& \int_{B(y, r)}\left(\phi\left(\left|u_{n}\right|\right) u_{n}^{2}\right)^{\tau}\left(\Phi_{*}^{\prime}\left(\left|u_{n}\right|\right)\left|u_{n}\right|\right)^{1-\tau} d x \\
& \leq\left(\int_{B(y, r)} \phi\left(\left|u_{n}\right|\right) u_{n}^{2} d x\right)^{\tau}\left(\int_{B(y, r)} \Phi_{*}^{\prime}\left(\left|u_{n}\right|\right)\left|u_{n}\right| d x\right)^{1-\tau} \\
& \leq p^{\tau} p^{*(1-\tau)}\left(\int_{B(y, r)} \Phi\left(\left|u_{n}\right|\right) d x\right)^{\tau}\left(\int_{B(y, r)} \Phi_{*}\left(\left|u_{n}\right|\right) d x\right)^{1-\tau} .
\end{aligned}
$$

Noting that $\left\{u_{n}\right\}$ is bounded in $W^{1} L_{\Phi}\left(\mathbb{R}^{N}\right)$, there exists a constant $M>0$ such that

$$
\begin{gathered}
\int_{\mathbb{R}^{N}} \Phi\left(\frac{\left|u_{n}\right|}{M}\right) d x \leq 1, \\
\int_{\mathbb{R}^{N}} \Phi\left(\frac{\left|\nabla u_{n}\right|}{M}\right) d x \leq 1 .
\end{gathered}
$$

Then, it follows from imbedding theorem (25) and Lemma 4 that

$$
\begin{aligned}
& \|u\|_{\Phi^{*}, B(y, r)} \\
& \quad \leq C_{1}\left(\|u\|_{\Phi, B(y, r)}+\|\nabla u\|_{\Phi, B(y, r)}\right) \\
& \quad \leq C_{2}\left(\left\|\frac{u}{M}\right\|_{\Phi, B(y, r)}+\left\|\frac{|\nabla u|}{M}\right\|_{\Phi, B(y, r)}\right) \\
& \quad \leq C_{2}\left[\left(\int_{B(y, r)} \Phi\left(\frac{|u|}{M}\right) d x\right)^{1 / p}+\left(\int_{B(y, r)} \Phi\left(\frac{|\nabla u|}{M}\right) d x\right)^{1 / p}\right],
\end{aligned}
$$

for some positive constants $C_{1}$ and $C_{2}$. Thus, by Lemma 5,

$$
\begin{aligned}
& \left(\int_{B(y, r)} \Phi_{*}\left(\left|u_{n}\right|\right) d x\right)^{1-\tau} \\
& \leq \zeta_{3}^{1-\tau}\left(\|u\|_{\Phi^{*}, B(y, r)}\right) \\
& \leq C_{3}\left[\zeta_{3}^{1-\tau}\left(\left(\int_{B(y, r)} \Phi\left(\frac{|u|}{M}\right) d x\right)^{1 / p}\right)\right. \\
& \left.+\zeta_{3}^{1-\tau}\left(\left(\int_{B(y, r)} \Phi\left(\frac{|\nabla u|}{M}\right) d x\right)^{1 / p}\right)\right] \\
& \leq C_{3}\left[\left(\int_{B(y, r)} \Phi\left(\frac{|u|}{M}\right) d x\right)^{q^{*}(1-\tau) / p}\right. \\
& \left.+\left(\int_{B(y, r)} \Phi\left(\frac{|\nabla u|}{M}\right) d x\right)^{q^{*}(1-\tau) / p}\right] \\
& \leq C_{4}\left[\left(\int_{B(y, r)} \Phi(|u|) d x\right)^{q^{*}(1-\tau) / p}\right. \\
& \left.+\left(\int_{B(y, r)} \Phi(|\nabla u|) d x\right)^{q^{*}(1-\tau) / p}\right],
\end{aligned}
$$

for some positive constants $C_{3}$ and $C_{4}$. Letting $1-\tau=p / q^{*}$, it follows from (58) and (61) that

$$
\begin{aligned}
& \int_{B(y, r)}\left(\phi\left(\left|u_{n}\right|\right) u_{n}^{2}\right)^{\tau}\left(\Phi_{*}^{\prime}\left(\left|u_{n}\right|\right)\left|u_{n}\right|\right)^{1-\tau} d x \\
& \quad \leq C\left(\int_{B(y, r)} \Phi\left(\left|u_{n}\right|\right) d x\right)^{\tau}\left[\int_{B(y, r)} \Phi(|u|) d x+\int_{B(y, r)} \Phi(|\nabla u|) d x\right],
\end{aligned}
$$


for some $C>0$. Then, coving $\mathbb{R}^{N}$ by balls of radius $r$, in such a way that each point of $\mathbb{R}^{N}$ is contained in at most $N+1$ balls, we have

$$
\begin{gathered}
\int_{\mathbb{R}^{N}}\left(\phi\left(\left|u_{n}\right|\right) u_{n}^{2}\right)^{\tau}\left(\Phi_{*}^{\prime}\left(\left|u_{n}\right|\right)\left|u_{n}\right|\right)^{1-\tau} d x \\
\leq(N+1) C \sup _{y \in \mathbb{R}^{N}}\left[\left(\int_{B(y, r)} \Phi\left(\left|u_{n}\right|\right) d x\right)^{\tau}\right. \\
\left.\cdot\left(\int_{\mathbb{R}^{N}} \Phi(|u|) d x+\int_{\mathbb{R}^{N}} \Phi(|\nabla u|) d x\right)\right] \longrightarrow 0
\end{gathered}
$$

as $n \longrightarrow \infty$. Noting that $\left\{u_{n}\right\}$ is bounded in $W^{1} L_{\Phi}\left(\mathbb{R}^{\mathrm{N}}\right)$, by (2.3), we get that $\int_{\mathbb{R}^{N}} \phi\left(\left|u_{n}\right|\right) u_{n}^{2} d x$ is a bounded sequence. If $\lambda>\tau$, letting $\mu=(\lambda-\tau) /(1-\tau)$, then

$$
\begin{aligned}
\int_{\mathbb{R}^{N}} g\left(\left|u_{n}\right|\right)\left|u_{n}\right| d x \\
\leq\left(\int_{\mathbb{R}^{N}} \phi\left(\left|u_{n}\right|\right) u_{n}^{2} d x\right)^{\mu} \\
\quad \cdot\left(\int_{\mathbb{R}^{N}}\left(\phi\left(\left|u_{n}\right|\right) u_{n}^{2}\right)^{\tau}\left(\Phi_{*}^{\prime}\left(\left|u_{n}\right|\right)\left|u_{n}\right|\right)^{1-\tau} d x\right)^{1-\mu} \longrightarrow 0
\end{aligned}
$$

as $n \longrightarrow \infty$. By imbedding theorem (25) and Lemma 7, one sees that $\int_{\mathbb{R}^{N}} \Phi_{*}^{\prime}\left(\left|u_{n}\right|\right)\left|u_{n}\right| d x$ is a bounded sequence. If $\lambda<\tau$, setting $\mu=(\tau-\lambda) / \tau$, then

$$
\begin{aligned}
\int_{\mathbb{R}^{N}} g\left(\left|u_{n}\right|\right)\left|u_{n}\right| d x \\
\leq\left(\int_{\mathbb{R}^{N}} \Phi_{*}^{\prime}\left(\left|u_{n}\right|\right)\left|u_{n}\right| d x\right)^{\mu} \\
\quad \cdot\left(\int_{\mathbb{R}^{N}}\left(\phi\left(\left|u_{n}\right|\right) u_{n}^{2}\right)^{\tau}\left(\Phi_{*}^{\prime}\left(\left|u_{n}\right|\right)\left|u_{n}\right|\right)^{1-\tau} d x\right)^{1-\mu} \longrightarrow 0
\end{aligned}
$$

as $n \longrightarrow \infty$. This ends the proof.

Theorem 19 (Vitali's convergence theorem [17]). Let $(X$, $\mathscr{A}, \mu)$ be a measure space and let $1 \leq p<\infty$. Let $\left\{f_{n}\right\}_{n=1}^{\infty}$ be a sequence in $L^{p}(X, \mathscr{A}, \mu)$ and let $f$ be an $\mathscr{A}$-measurable function such that $f$ is finite $\mu$-a.e. and $f_{n} \longrightarrow f \mu$-a.e. Then, $f \in L^{p}(X, \mathscr{A}, \mu)$ and $\left\|f-f_{n}\right\|_{p} \longrightarrow 0$ if and only if

(i) For each $\varepsilon>0$, there exists a set $A_{\varepsilon} \in \mathscr{A}$ such that $\mu\left(A_{\varepsilon}\right)<\infty$ and $\int_{A_{\varepsilon}^{c}}\left|f_{n}\right|^{p} d \mu<\varepsilon$ for all $n \in \mathbb{R}^{N}$

(ii) $\lim _{\mu(E) \longrightarrow 0} \int_{E}\left|f_{n}\right|^{p} d \mu=0$

uniformly in $n$, i.e., for each $\varepsilon>0$, there is a $\delta>0$ such that $E \in \mathscr{A}$ and $\mu(E)<\delta$ imply $\int_{E}\left|f_{n}\right|^{p} d \mu<\varepsilon$ for all $n \in \mathbb{N}$.

\section{Main Result}

Theorem 20. Suppose conditions $\left(\phi_{1}\right)-\left(\phi_{3}\right)$ and $\left(f_{1}\right)-\left(f_{5}\right)$ hold. Then, (1) has a nonnegative weak solution.

Proof. By Lemma 16, there exists a sequence $\left\{u_{n}\right\} \subset A$, such that

$$
I\left(u_{n}\right) \longrightarrow I_{0}, I^{\prime}\left(u_{n}\right) \longrightarrow 0 \operatorname{in}\left(W^{1} L_{\Phi}\left(\mathbb{R}^{N}\right)\right)^{*}
$$

Step 1. There exists some constant $C>0$ such that $\left\|u_{n}\right\|_{1, \Phi} \leq$ C. By (66) and (7), one has

$$
\begin{aligned}
I_{0}+o_{n}(1)= & \int_{\mathbb{R}^{N}} \Phi\left(\left|u_{n}\right|\right)+\Phi\left(\left|\nabla u_{n}\right|\right) d x \\
& -\frac{1}{p+\theta} \int_{\mathbb{R}^{N}} \phi\left(\left|u_{n}\right|\right) u_{n}^{2}+\phi\left(\left|\nabla u_{n}\right|\right)\left|\nabla u_{n}\right|^{2} d x \\
& +\int_{\mathbb{R}^{N}} \frac{1}{p+\theta} f\left(u_{n}\right) u_{n}-F\left(u_{n}\right) d x \\
\geq & \left(1-\frac{p}{p+\theta}\right) \int_{\mathbb{R}^{N}} \Phi\left(\left|u_{n}\right|\right)+\Phi\left(\left|\nabla u_{n}\right|\right) d x \\
& +\int_{\mathbb{R}^{N}} \frac{1}{p+\theta} f\left(\left|u_{n}\right|\right)\left|u_{n}\right|-F\left(\left|u_{n}\right|\right) d x \\
\geq & \left(1-\frac{p}{p+\theta}\right) \int_{\mathbb{R}^{N}} \Phi\left(\left|u_{n}\right|\right)+\Phi\left(\left|\nabla u_{n}\right|\right) d x .
\end{aligned}
$$

In the last step, we use condition $\left(f_{5}\right)$. Hence, by Lemma 4 , there exists some constant $C>0$ such that $\left\|u_{n}\right\|_{1, \Phi} \leq C$.

Step 2. There exist $R>0, \alpha>0$, and $\left\{y_{n}\right\} \subset \mathbb{R}^{N}$, such that

$$
\int_{y_{n}+B_{R}} \Phi\left(\left|u_{n}\right|\right) d x \geq \alpha>0
$$

By Step $1, \Phi\left(\left|u_{n}\right|\right)$ is bounded in $L^{1}\left(\mathbb{R}^{N}\right)$. Lemma 17 shows that one of two situations (vanishing and nonvanishing) occur. We need only to show that the vanishing case is impossible. If vanishing occurs, by Lemma 18, $\lim _{n \rightarrow \infty} \int_{\mathbb{R}^{N}}$ $g\left(\left|u_{n}\right|\right)\left|u_{n}\right| d x=0$. It follows from (29) and (30) that

$$
\lim _{n \longrightarrow \infty} \int_{\mathbb{R}^{N}} f\left(\left|u_{n}\right|\right)\left|u_{n}\right| d x=\lim _{n \longrightarrow \infty} \int_{\mathbb{R}^{N}} F\left(\left|u_{n}\right|\right) d x=0
$$

Notice $u_{n} \in A$, we obtain

$$
\begin{aligned}
0 & <I_{0} \\
& \leq \limsup _{n \longrightarrow \infty}\left[\frac{1}{q} \int_{\mathbb{R}^{N}} \phi\left(\left|u_{n}\right|\right) u_{n}^{2}+\phi\left(\left|\nabla u_{n}\right|\right)\left|\nabla u_{n}\right|^{2} d x-\int_{\mathbb{R}^{N}} F\left(\left|u_{n}\right|\right) d x\right] \\
& =\limsup _{n \rightarrow \infty} \int_{\mathbb{R}^{N}} \frac{1}{q} f\left(\left|u_{n}\right|\right)\left|u_{n}\right|-F\left(\left|u_{n}\right|\right) d x=0 .
\end{aligned}
$$


This is a contradiction. Hence, only nonvanishing is possible, i.e., there exist $R>0, \alpha>0$, and $\left\{y_{n}\right\} \subset \mathbb{R}^{N}$, such that

$$
\int_{y_{n}+B_{R}} \Phi\left(\left|u_{n}\right|\right) d x \geq \alpha>0
$$

Step 3. There exists $\bar{u} \in W^{1} L_{\Phi}\left(\mathbb{R}^{N}\right)$, such that $\nabla \bar{u}_{n} \longrightarrow \nabla \overline{\mathrm{u}}$ a.e. in $\mathbb{R}^{N}$, where $\bar{u}_{n}(x)=u_{n}\left(x+y_{n}\right)$.

For any $\psi \in W^{1} L_{\Phi}\left(\mathbb{R}^{N}\right)$, set $\psi_{n}(x)=\psi\left(x-y_{n}\right)$. Then, $\|\psi\|_{1, \Phi}=\left\|\psi_{n}\right\|_{1, \Phi}$, and

$$
\begin{aligned}
\left|\left\langle I^{\prime}\left(\bar{u}_{n}\right), \psi\right\rangle\right| & =\left|\left\langle I^{\prime}\left(u_{n}\right), \psi_{n}\right\rangle\right| \\
& \leq\left\|I^{\prime}\left(u_{n}\right)\right\|_{\left(W^{1} L_{\Phi}\left(\mathbb{R}^{N}\right)\right)^{*}}\left\|\psi_{n}\right\|_{1, \Phi} \\
& =\left\|I^{\prime}\left(u_{n}\right)\right\|_{\left(W^{1} L_{\Phi}\left(\mathbb{R}^{N}\right)\right)^{*}}\|\psi\|_{1, \Phi} \longrightarrow 0,
\end{aligned}
$$

as $n \longrightarrow \infty$. Since $\left\|\bar{u}_{n}\right\|_{1, \Phi}=\left\|\bar{u}_{n}\right\|_{1, \Phi}$, so $\left\{\bar{u}_{n}\right\}$ is a bounded sequence in $W^{1} L_{\Phi}\left(\mathbb{R}^{N}\right)$. Hence, there exists $\bar{u} \in W^{1} L_{\Phi}\left(\mathbb{R}^{N}\right)$ and $\bar{u} \equiv 0$ such that some sequence of $\left\{\bar{u}_{n}\right\}$, still denoted by $\left\{\bar{u}_{n}\right\}$,

$$
\left\{\begin{array}{l}
\bar{u}_{n} \rightarrow \bar{u} \text { in } W^{1} L_{\Phi}\left(\mathbb{R}^{N}\right), \\
\bar{u}_{n} \longrightarrow \bar{u} \text { a.e.in } \mathbb{R}^{N}, \\
\int_{\Omega} g\left(\left|\bar{u}_{n}-\bar{u}\right|\right)\left|\bar{u}_{n}-\bar{u}\right| d x \longrightarrow 0 \text { for any bounded subset } \Omega \subset \mathbb{R}^{N} .
\end{array}\right.
$$

Let $\eta_{r} \in C_{0}^{\infty}\left(\mathbb{R}^{N}\right)$ such that $0 \leq \eta \leq 1$, with $\eta_{r}=1$ in $B_{r}=$ $\left\{x \in \mathbb{R}^{N}|| x \mid \leq r\right\}, \eta_{r}=0$ in $B_{2 r}^{c}$, and $\left|\nabla \eta_{r}\right| \leq C / r$ for some $C>0$. Then, it is easy to see that

$$
\left\langle I^{\prime}\left(\bar{u}_{n}\right)-I^{\prime}(\bar{u}),\left(\bar{u}_{n}-\bar{u}\right) \eta_{r}\right\rangle=o_{n}(1) .
$$

On the other hand,

$$
\begin{aligned}
& \left\langle I^{\prime}\left(\bar{u}_{n}\right)-I^{\prime}(\bar{u}),\left(\bar{u}_{n}-\bar{u}\right) \eta_{r}\right\rangle \\
& =\int_{B_{2 r}} \eta_{r}\left(\phi\left(\left|\bar{u}_{n}\right|\right) \bar{u}_{n}-\phi(|\bar{u}|) \bar{u}\right)\left(\bar{u}_{n}-\bar{u}\right) d x \\
& \quad+\int_{B_{2 r}} \eta_{r}\left(\phi\left(\left|\nabla \bar{u}_{n}\right|\right) \nabla \bar{u}_{n}-\phi(|\nabla \bar{u}|) \nabla \bar{u}\right) \cdot \nabla\left(\bar{u}_{n}-\bar{u}\right) d x \\
& \quad+\int_{B_{2 r} \backslash B_{r}}\left(\phi\left(\left|\nabla \bar{u}_{n}\right|\right) \nabla \bar{u}_{n}-\phi(|\nabla \bar{u}|) \nabla \bar{u}\right) \cdot \nabla \eta_{r}\left(\bar{u}_{n}-\bar{u}\right) d x \\
& \quad-\int_{B_{2 r}} \eta_{r}\left(f\left(\bar{u}_{n}\right)-f(\bar{u})\right)\left(\bar{u}_{n}-\bar{u}\right) d x .
\end{aligned}
$$

By (17), (26), and Lemma 4,

$$
\begin{aligned}
\left|\int_{B_{2 r} \mid B_{r}}\left(\phi\left(\nabla \bar{u}_{n}\right) \nabla \bar{u}_{n}-\phi(\nabla \bar{u}) \nabla \bar{u}\right) \cdot \nabla \eta_{r}\left(\bar{u}_{n}-\bar{u}\right) d x\right| \\
\leq \frac{C}{r}\left\|\left|\phi\left(\nabla \bar{u}_{n}\right) \nabla \bar{u}_{n}-\phi(\nabla \bar{u}) \nabla \bar{u}\right|\right\|_{\tilde{\Phi}, B_{2 r} \backslash B_{r}}\left\|\bar{u}_{n}-\bar{u}\right\|_{\Phi, B_{2 r} \backslash B_{r}} \\
\leq \frac{C}{r}\left[\zeta_{4}^{-1}\left(\int_{B_{2 r} \backslash B_{r}} \Phi\left(\nabla \bar{u}_{n}\right) d x\right)+\zeta_{4}^{-1}\left(\int_{B_{2 r} \backslash B_{r}} \Phi(\nabla \bar{u}) d x\right)\right] \\
\cdot\left[\zeta_{0}^{-1}\left(\int_{B_{2 r} \mid B_{r}} \Phi\left(\bar{u}_{n}\right) d x\right)+\zeta_{0}^{-1}\left(\int_{B_{2 r} \mid B_{r}} \Phi(\bar{u}) d x\right)\right] .
\end{aligned}
$$

Noting that $\bar{u}_{n}$ is bounded in $W^{1} L_{\Phi}\left(\mathbb{R}^{N}\right)$, for any $\varepsilon>$ 0 , there exists $r_{0}>0$ such that for $r>r_{0}$,

$$
\left|\int_{B_{2 r} \backslash B_{r}}\left(\phi\left(\nabla \bar{u}_{n}\right) \nabla \bar{u}_{n}-\phi(\nabla \bar{u}) \nabla \bar{u}\right) \cdot \nabla \eta_{r}\left(\bar{u}_{n}-\bar{u}\right) d x\right| \leq \varepsilon .
$$

For fix $r$ and $\delta>0$, by the Egoroff theorem, there exists $E_{\delta} \subset B_{2 r}$ such that $m\left(E_{\delta}\right)<\delta$, and $\left(f\left(\bar{u}_{n}\right)-f(\bar{u})\right)$ $\left(\bar{u}_{n}-\bar{u}\right) \longrightarrow 0$ uniformly on $B_{2 r} \backslash E_{\delta}$ as $n \longrightarrow+\infty$. Thus, for any $\varepsilon>0, \delta>0$, there exists $n_{0}>0$ such that, for $n \geq n_{0}$,

$$
\left|\int_{B_{2 r} \backslash E_{\delta}} \eta_{r}\left(f\left(\bar{u}_{n}\right)-f(\bar{u})\right)\left(\bar{u}_{n}-\bar{u}\right) d x\right| \leq \varepsilon .
$$

By $\left(f_{2}\right)$ and $\left(f_{3}\right)$, there exists some $C>0$ such that

$$
f(t) \leq\left\{\begin{array}{l}
C t \phi(t) \text { for } 0<t<1, \\
C g(t) \text { for } t \geq 1 .
\end{array}\right.
$$

Let $1<\mu<q^{*} /\left(p \lambda+(1-\lambda) q^{*}\right)$ be a fixed constant. Then, by Lemma 4 , Lemma 5 , and Lemma 7 , for $t \geq 1$,

$$
\begin{aligned}
(t f(t))^{\mu} & \leq C\left(t^{2} \phi(t)\right)^{\lambda \mu}\left(t \Phi_{*}^{\prime}(t)\right)^{(1-\lambda) \mu} \\
& \leq C(\Phi(t))^{\lambda \mu}\left(\Phi_{*}(t)\right)^{(1-\lambda) \mu} \leq C t^{\lambda \mu p}\left(\Phi_{*}(t)\right)^{(1-\lambda) \mu} \\
& \leq C\left(\Phi_{*}(t)\right)^{\lambda \mu p / q^{*}+(1-\lambda) \mu} \leq C \Phi_{*}(t),
\end{aligned}
$$

for some $C>0$. Set $E_{\delta, 1}=\left\{x \in E_{\delta}|| \bar{u}(x) \mid<1\right\}$, and $E_{\delta, 2}=$ $E_{\delta} \backslash E_{\delta, 1}$. Using Hölder inequality, we obtain

$$
\begin{aligned}
& \left|\int_{E_{\delta}} \eta_{r}\left(f\left(\bar{u}_{n}\right)-f(\bar{u})\right)\left(\bar{u}_{n}-\bar{u}\right) d x\right| \\
& \quad \leq C\left(\int_{E_{\delta}}\left|\left(f\left(\bar{u}_{n}\right)-f(\bar{u})\right)\left(\bar{u}_{n}-\bar{u}\right)\right|^{\mu} d x\right)^{1 / \mu}\left(m\left(E_{\delta}\right)\right)^{1-1 / \mu} \\
& \quad \leq C\left(\int_{E_{\delta}}\left|f\left(\bar{u}_{n}\right) \bar{u}_{n}\right|^{\mu}+|f(\bar{u}) \bar{u}|^{\mu} d x\right)^{1 / \mu} \delta^{1-1 / \mu} .
\end{aligned}
$$


Since $u_{n}$ and $u$ is bounded in $W^{1} L_{\Phi}\left(\mathbb{R}^{N}\right)$, so there exists a constant $C>0$ such that

$$
\int_{\mathbb{R}^{N}} \Phi_{*}\left(\left|u_{n}\right|\right) d x \leq C, \int_{\mathbb{R}^{N}} \Phi_{*}(|u|) d x \leq C .
$$

Therefore, for some $C>0$,

$$
\begin{gathered}
\int_{E_{\delta}}|f(\bar{u}) \bar{u}|^{\mu} d x \leq C \int_{E_{\delta, 1}}\left(\bar{u}^{2} \phi(|\bar{u}|)\right)^{\mu} d x+C \int_{E_{\delta, 2}} \Phi_{*}(|\bar{u}|) d x \leq C, \\
\int_{E_{\delta}}\left|f\left(\bar{u}_{n}\right) \bar{u}_{n}\right|^{\mu} d x \leq C \int_{E_{\delta, 1}}\left(\bar{u}_{n}^{2} \phi\left(\left|\bar{u}_{n}\right|\right)\right)^{\mu} d x+C \int_{E_{\delta, 2}} \Phi_{*}\left(\left|\bar{u}_{n}\right|\right) d x \leq C .
\end{gathered}
$$

Choosing $\delta>0$ small enough, one has

$$
\left|\int_{E_{\delta}} \eta_{r}\left(f\left(\bar{u}_{n}\right)-f(\bar{u})\right)\left(\bar{u}_{n}-\bar{u}\right) d x\right| \leq \varepsilon .
$$

Hence, such that for $n>n_{0}$,

$$
\left|\int_{B_{2 r}} \eta_{r}\left(f\left(\left|\bar{u}_{n}\right|\right)-f(|\bar{u}|)\right)\left(\bar{u}_{n}-\bar{u}\right) d x\right| \leq 2 \epsilon .
$$

Since $\left(\phi\left(\left|\bar{u}_{n}\right|\right) \bar{u}_{n}-\phi(|\bar{u}|) \bar{u}\right)\left(\bar{u}_{n}-\bar{u}\right) \geq 0$ and $\left(\phi\left(\left|\nabla \bar{u}_{n}\right|\right) \nabla\right.$ $\left.\bar{u}_{n}-\phi(|\nabla \bar{u}|) \nabla \bar{u}\right) \cdot \nabla\left(\bar{u}_{n}-\bar{u}\right) \geq 0$, it follows from (74)-(85) that for any $\epsilon>0$, there exist $r_{0}$ and $n_{1} \geq n_{0}$ such that for $n>n_{1}$ and $r \geq r_{0}$,

$$
\int_{B_{r}}\left(\phi\left(\left|\nabla \bar{u}_{n}\right|\right) \nabla \bar{u}_{n}-\phi(|\nabla \bar{u}|) \nabla \bar{u}\right) \cdot \nabla\left(\bar{u}_{n}-\bar{u}\right) d x \leq C \epsilon .
$$

It follows that, passing to a subsequence if necessary, $\left(\phi\left(\left|\nabla \bar{u}_{n}\right|\right) \nabla \bar{u}_{n}-\phi(|\nabla \bar{u}|) \nabla \bar{u}\right) \cdot \nabla\left(\bar{u}_{n}-\bar{u}\right) \longrightarrow 0 \quad$ a.e. in $B_{r}$. Using Lemma 6 [18], we get that

$$
\nabla \bar{u}_{n} \longrightarrow \nabla \bar{u} \text { a.e.in } B_{r} .
$$

Since $r$ is arbitrary, one can see that $\nabla \bar{u}_{n} \longrightarrow \nabla \bar{u}$ a.e. in $\mathbb{R}^{N}$.

Step 4. $I^{\prime}(\bar{u})=0, \bar{u} \in A$.

We first show that $\phi\left(\left|\nabla \overline{\mathcal{u}}_{n}\right|\right) \nabla \bar{u}_{n} \rightarrow \phi(|\nabla \bar{u}|) \nabla \bar{u}$ in $L_{\tilde{\Phi}}\left(\mathbb{R}^{N}\right)$, i.e., for any $\psi \in L_{\Phi}\left(\mathbb{R}^{N}\right)$,

$$
\lim _{n \longrightarrow \infty} \int_{\mathbb{R}^{N}} \phi\left(\left|\nabla \bar{u}_{n}\right|\right)\left|\nabla \bar{u}_{n}\right| \psi d x=\int_{\mathbb{R}^{N}} \phi(|\nabla \bar{u}|)|\nabla \bar{u}| \psi d x .
$$

Since $\nabla \bar{u}_{n}$ is bounded in $W^{1} L_{\Phi}\left(\mathbb{R}^{N}\right)$, by (26), there exists some $M>0$ such that

$$
\left\|\phi\left(\left|\nabla \bar{u}_{n}\right|\right)\left|\nabla \bar{u}_{n}\right|\right\|_{\tilde{\Phi}} \leq M
$$

Then, for any $\epsilon>0$, there exist $r>0$ and $\delta>0$ such that for any $m(E) \leq \delta$,

$$
\begin{gathered}
\int_{B_{r}^{c}} \phi\left(\left|\nabla \bar{u}_{n}\right|\right)\left|\nabla \bar{u}_{n}\right| \psi d x \leq 2|| \phi\left(\left|\nabla \bar{u}_{n}\right|\right)\left|\nabla \bar{u}_{n}\right|\left\|{ }_{\tilde{\Phi}}\right\| \psi \|_{\Phi, B_{r}^{c}} \leq \epsilon \\
\int_{E} \phi\left(\left|\nabla \bar{u}_{n}\right|\right)\left|\nabla \bar{u}_{n}\right| \psi d x \leq 2\left\|\phi\left(\left|\nabla \bar{u}_{n}\right|\right)\left|\nabla \bar{u}_{n}\right|\right\|_{\tilde{\Phi}}\|\psi\|_{\Phi, E} \leq \epsilon .
\end{gathered}
$$

Then, by Theorem 19,

$$
\phi\left(\nabla \bar{u}_{n}\right) \nabla \bar{u}_{n}-\phi(|\nabla \bar{u}|)|\nabla \bar{u}| \text { in }\left(W^{1} L_{\Phi}\left(\mathbb{R}^{N}\right)\right)^{*} .
$$

Similarly, we can have

$$
\phi\left(\left|\bar{u}_{n}\right|\right) \bar{u}_{n} \rightarrow \phi(|\bar{u}|) \bar{u}, f\left(\bar{u}_{n}\right) \rightarrow f(\bar{u}), \text { in }\left(W^{1} L_{\Phi}\left(\mathbb{R}^{N}\right)\right)^{*} .
$$

It follows from (91) and (92) that for any $\psi \in W^{1} L_{\Phi}\left(\mathbb{R}^{N}\right)$,

$$
0=\lim _{n \longrightarrow \infty}\left\langle I^{\prime}\left(\bar{u}_{n}\right), \psi\right\rangle=\lim _{n \longrightarrow \infty}\left\langle I^{\prime}(\bar{u}), \psi\right\rangle .
$$

In particular, taking $\psi=\bar{u}$, we get that $\left\langle I^{\prime}(\bar{u}), \bar{u}\right\rangle=0$. Since $\bar{u} \equiv 0$, so $\bar{u} \in A$.

Step 5. $I(\bar{u})=I_{0}$.

By the definition of $\bar{u}_{k}$, we obtain that

$$
I\left(\bar{u}_{n}\right)=I\left(u_{n}\right),\left\langle I^{\prime}\left(\bar{u}_{n}\right), \bar{u}_{n}\right\rangle=\left\langle I^{\prime}\left(u_{n}\right), u_{n}\right\rangle=o_{n}(1) .
$$

Hence, by (7), condition $\left(f_{5}\right)$, and Fatou's lemma,

$$
\begin{aligned}
I(\bar{u}) \geq & I_{0}=\lim _{n \longrightarrow \infty} I\left(\bar{u}_{n}\right) \\
= & \lim _{n \longrightarrow \infty}\left[\int_{\mathbb{R}^{N}} \Phi\left(\left|\bar{u}_{n}\right|\right)+\Phi\left(\left|\nabla \bar{u}_{n}\right|\right) d x-\int_{\mathbb{R}^{N}} F\left(\bar{u}_{n}\right) d x\right] \\
= & \lim _{n \longrightarrow \infty}\left[\int_{\mathbb{R}^{N}} \Phi\left(\left|\bar{u}_{n}\right|\right)+\Phi\left(\left|\nabla \bar{u}_{n}\right|\right)-\frac{1}{p} \phi\left(\left|\bar{u}_{n}\right|\right) \bar{u}_{n}^{2}\right. \\
& \left.-\frac{1}{p} \phi\left(\left|\nabla \bar{u}_{n}\right|\right)\left|\nabla \bar{u}_{n}\right|^{2} d x+\int_{\mathbb{R}^{N}} \frac{1}{p} f\left(\bar{u}_{n}\right)-F\left(\bar{u}_{n}\right) d x\right] \\
\geq & {\left[\int_{\mathbb{R}^{N}} \Phi(|\bar{u}|)+\Phi(|\nabla \bar{u}|)-\frac{1}{p} \phi(|\bar{u}|) \bar{u}^{2}\right.} \\
& \left.-\frac{1}{p} \phi(|\nabla \bar{u}|)|\nabla \bar{u}|^{2} d x+\int_{\mathbb{R}^{N}} \frac{1}{p} f(\bar{u}) \bar{u}-F(\bar{u}) d x\right] \\
= & {\left[\int_{\mathbb{R}^{N}} \Phi(|\bar{u}|)+\Phi(|\nabla \bar{u}|) d x-\int_{\mathbb{R}^{N}} \frac{1}{p} F(\bar{u}) d x\right]=I(\bar{u}) . }
\end{aligned}
$$

It follows that $I(\bar{u})=I_{0}$. By $\left(f_{1}\right)$, we get that $\bar{u}^{-}=\max$ $\{-\bar{u}, 0\}=0$. Hence, $\bar{u} \geq 0$. This ends the proof. 


\section{Data Availability}

The data used to support the findings of this study are included within the article.

\section{Conflicts of Interest}

The authors declare that they have no conflicts of interest.

\section{Acknowledgments}

This work is supported by the NSFC (11571339, 11871195, and 11301153), the Key Scientific Research Projects of Higher Education Institutions in Henan Province (20B110004), and the doctoral researcher foundation of Henan University of Science and Technology (13480051).

\section{References}

[1] Z. Guo, "On the number of positive solutions for quasilinear elliptic eigenvalue problems," Nonlinear Analysis, vol. 27, no. 2, pp. 229-247, 1996.

[2] Z. Guo and J. R. L. Webb, "Large and small solutions of a class of quasi-linear eigenvalue problems," Journal of Differential Equations, vol. 180, no. 1, pp. 1-50, 2002.

[3] M. Guedda and L. Veron, "Bifurcation phenomena associated to the p-Laplace operator," Transactions of the American Mathematical Society, vol. 310, pp. 419-431, 1988.

[4] Z. Zhang and S. Li, "On sign-changing and multiple solutions of the p-Laplacian," Journal of Functional Analysis, vol. 197, no. 2, pp. 447-468, 2003.

[5] Z. Zhang, J. Chen, and S. Li, "Construction of pseudo-gradient vector field and sign-changing multiple solutions involving p-Laplacian," Journal of Differential Equations, vol. 201, no. 2, pp. 287-303, 2004.

[6] J.-P. Gossez, "Nonlinear elliptic boundary value problems for equations with rapidly (or slowly) increasing coefficients," Transactions of the American Mathematical Society, vol. 190, pp. 163-205, 1974.

[7] Z. Guo and Z. Zhang, " $W^{1, p}$ versus $C^{1}$ local minimizers and multiplicity results for quasilinear elliptic equations," Journal of Mathematical Analysis and Applications, vol. 286, no. 1, pp. 32-50, 2003.

[8] J. Su and R. Tian, "Weighted Sobolev embeddings and radial solutions of inhomogeneous quasi-linear elliptic equations," Communications on Pure \& Applied Analysis, vol. 9, no. 4, pp. 885-904, 2010.

[9] G. Li and Z. Guo, "Multiple solutions for the p\&q-Laplacian problem with critical exponent," Acta Mathematica Scientia, vol. 29, no. 4, pp. 903-918, 2009.

[10] G. Li and X. Liang, "The existence of nontrivial solutions to nonlinear elliptic equation of p-q- Laplacian type on $\mathbb{R}^{N}$," Nonlinear Analysis: Theory, Methods \& Applications, vol. 71, no. 5-6, pp. 2316-2334, 2009.

[11] B. Franchi, E. Lanconelli, and J. Serrin, "Existence and uniqueness of nonnegative solutions of quasilinear equations in $\mathrm{R}^{\mathrm{n}}$ ," Adv. Math., vol. 118, no. 2, pp. 177-243, 1996.

[12] E. L. M. Carvalho, E. D. da Silva, and C. Goulart, "Quasi-linear elliptic problems with concave-convex nonlinearities," Communications in Contemporary Mathematics, vol. 19, pp. 125,2017
[13] E. L. M. Carvalho, E. D. da Silva, J. V. Goncalves, and C. Goulart, "Critical quasi-linear elliptic problems using concave-convex nonlinearities," Annali di Matematica Pura ed Applicata (1923-), vol. 198, pp. 693-726, 2019.

[14] R. A. Adams and J. F. Fournier, Sobolev Spaces, Academic press, 2nd edition, 2003.

[15] N. Fukagai, M. Ito, and K. Narukawa, "Positive solutions of quasilinear elliptic equations with critical Orlicz-Sobolev nonlinearity on $\mathbb{R}^{N}$," Funkcialaj Ekvacioj, vol. 49, no. 2, pp. 235267,2006

[16] K. C. Chang, Critical Point Theory and Its Application, Shanghai Sci. Tech. Press, 1986.

[17] E. Hewitt and K. Stromberg, Real and Abstract Analysis, Springer-Verlag, 3rd edition, 1975.

[18] G. Dal Maso and F. Murat, "Almost everywhere convergence of gradients of solutions to nonlinear elliptic systems," Nonlinear Analysis, vol. 31, no. 3-4, pp. 405-412, 1998. 